\title{
Experimental study GW23-e0999 CDP-CHOLINE IMPROVES THE OUTCOME OF CARDIAC ARREST VERSUS EPINEPHRINE IN RATS
}

doi:10.1136/heartjnl-2012-302920b.1

Huang Yu, He Qing, He Qing. Emergency Department, West China Hospital of Sichuan University, Chengdu, China

Objectives CDP-Choline is a cholinergic agent which can both stimulate the cholinergic pathway and increase blood pressure. We aimed to investigate the effects of CDP-Choline on the outcome of cardiac arrest in comparison to epinephrine.

Methods Cardiac arrest was induced by asphyxia in 45 rats. After 7 min untreated cardiac arrest, resuscitation was attempted. The rats were allocated to different groups, each group treated by $2 \mathrm{ml} /$ $\mathrm{kg}$ saline, $100 \mu \mathrm{g} / \mathrm{kg}$ epinephrine, $250 \mathrm{mg} / \mathrm{kg}$ CDP-Choline. The hemodynamic parameters reflecting cardiac function were monitored for $2 \mathrm{~h}$ after resuscitation and the hearts were harvested for ultrastructural observation at the end of monitoring.

Results Both epinephrine and CDP-Choline improved the blood pressure during CPR and the rate of return of spontaneous circulation (ROSC). The $\mathrm{dP} / \mathrm{dtmax}$ and the absolute value $-\mathrm{dP} / \mathrm{dtmax}$ was significantly decreased in all groups after resuscitation. However, postresuscitation cardiac function in CDP-Choline group and placebo group was better than in epinephrine group. The dP/ dtmax and the absolute value of $-\mathrm{dP} / \mathrm{dtmax}$ began to be higher with CDP-Choline and saline compared with epinephrine. The sections of the left ventricular anterior wall tissue were observed by the electron microscopy. We could find myocardium injured severely when treated with epinephrine. In epinephrine group, oedematous myofilaments, and oedematous mitochondria with destructed crista, were observed in cardiomyocytes. Compared to epinephrine group, less myocardial injury was observed by electron microscopy in CDP-Choline and placebo groups.

Conclusions The first finding of our investigation is that CDP-Choline had advantages similar to epinephrine in ROSC and MAP during CPR. This indicates that the beneficial effects of CDP-Choline in the improvement of ROSC may be related to its vasopressor effect. Moreover, for the negative inotropic and chronotropic effects of CDPCholine, we strongly suggested that certain inotropic or chronotropic effects would not compromise initial resuscitation. The second finding of our investigation is that epinephrine had worsen the postresuscitation myocardial dysfunction and acute myocardial I/R injury according to the hemodynamic and cardiomyocytes microstructure observation. On the other hand, CDP-Choline hadn't worsen postresuscitation myocardial dysfunction and acute myocardial I/R injury compared to saline, although it would stimulate the adrenergic system and increase blood pressure. Previous investigations have demonstrated that cholinergic stimulation has important protection effects on myocardium during acute I/R injury, besides, CDP-Choline may also decrease myocardial oxygen requirements by peripheral cholinergic stimulation. Combining these together, it is conceivable that the cholinergic protection effect of CDP-Choline on myocardium during I/R injury may play an important role in its effects on $\mathrm{CPR}$, and may produce rather better effects than epinephrine.

CDP-Choline increases the rate of ROSC when given during resuscitation, and it wouldn't increase the severity of postresuscitation myocardial dysfunction while epinephrine appears to be harmful to postresuscitation myocardial function. 